\title{
Modifying Pivot Elements in Gaussian Elimination*
}

\author{
By G. W. Stewart
}

\begin{abstract}
The rounding-error analysis of Gaussian elimination shows that the method is stable only when the elements of the matrix do not grow excessively in the course of the reduction. Usually such growth is prevented by interchanging rows and columns of the matrix so that the pivot element is acceptably large. In this paper the alternative of simply altering the pivot element is examined. The alteration, which amounts to a rank one modification of the matrix, is undone at a later stage by means of the well-known formula for the inverse of a modified matrix. The technique should prove useful in applications in which the pivoting strategy has been fixed, say to preserve sparseness in the reduction.
\end{abstract}

1. Introduction. Let $A$ be a real matrix of order $n$. The method of Gaussian elimination may be regarded as a technique for computing the $L U$ decomposition of $A$ into the product of a unit lower triangular matrix $L$ and an upper triangular matrix $U$. Specifically, at the $k$ th step of the reduction, we have

$$
A=\left(\begin{array}{ll}
L_{11}^{(k)} & 0 \\
L_{21}^{(k)} & I
\end{array}\right)\left(\begin{array}{cc}
U_{11}^{(k)} & U_{12}^{(k)} \\
0 & A_{22}^{(k)}
\end{array}\right),
$$

where $L_{11}{ }^{(k)}$ and $U_{11}{ }^{(k)}$ are of order $k$. The $(k+1)$ th row of $U$ is then given by

$$
u_{k+1, i}=a_{k+1, i}^{(k)} \quad(j=k+1, k+2, \cdots, n),
$$

the $(k+1)$ th column of $L$ by

$$
l_{i, k+1}=a_{i, k+1}^{(k)} / a_{k+1, k+1}^{(k)} \quad(i=k+1, k+2, \cdots, n),
$$

and the matrix $A_{22}{ }^{(k+1)}$ by

$$
a_{i i}^{(k+1)}=a_{i i}^{(k)}-l_{i, k+1} u_{k+1, j} \quad(i, j=k+2, \cdots, n) .
$$

The element $a_{k+1, k+1}{ }^{(k)}$ is called a pivot element for the algorithm. If it is zero, the algorithm fails, and if it is too small, the algorithm becomes unstable in the presence of rounding errors. Usually, this problem is avoided by interchanging two rows and perhaps two columns of $A_{22}{ }^{(k)}$ to bring an acceptably large element into the pivot position. However, in applications involving large sparse matrices, an unhappy pivot selection may destroy the sparsity of the subsequent matrices. Indeed in some applications the choice of pivots is determined entirely from the sparsity structure of $A$, leaving no freedom to pivot for stability (e.g. see [1]).

In this paper we shall examine the technique of modifying the pivot element

Received April 19, 1973.

AMS (MOS) subject classifications (1970). Primary 65F05.

* This work was supported in part by the Office of Naval Research under Contract No. N0001467-A-0314-0018. 
so that it is acceptably large and then undoing the modification later after the $L U$ decomposition of the modified matrix has been computed. Since the emergence of a small pivot element in Gaussian elimination betokens a numerical ill-determination of the $L U$ decomposition, we shall not try to obtain the $L U$ decomposition of $A$ itself; rather, we shall show how the $L U$ decomposition of the modified matrix may be used to solve linear systems involving $A$.

The next section will be devoted to describing the mechanics of the technique. The effects of rounding error will be discussed in Section 3.

2. Modifying Pivot Elements in the Solution of Linear Equations. In this section we shall show how the solution of the equation

$$
A x=b
$$

can be obtained from the solution of

$$
B y=b,
$$

where $A$ and $B$ differ only in their $(1,1)$-elements. We shall then indicate the applications of this technique in Gaussian elimination.

Since $A$ and $B$ differ only in their $(1,1)$-elements, $B$ can be written in the form

$$
B=A+\sigma e_{1} e_{1}^{T},
$$

where $e_{1}$ is the first column of the identity matrix. Then it follows from the wellknown modification formula (see [2, p. 123]) that

$$
A^{-1}=B^{-1}-\tau B^{-1} e_{1} e_{1}^{T} B^{-1},
$$

where

$$
\tau=1 /\left(e_{1}{ }^{T} B^{-1} e_{1}-\sigma^{-1}\right) .
$$

Since $x=A^{-1} b$ we have from (2.2) and (2.3),

$$
x=y-\tau B^{-1} e_{1} e_{1}^{T} y=y-\tau y_{1} c_{1},
$$

where $y_{1}$ is the first component of $y$ and $c_{1}$ is the first column of $B^{-1}$. Thus, the solution of (2.1) can be obtained from the solution of (2.2) by subtracting a suitable correction vector.

The economics of this technique are favorable. The system (2.2) costs no more to solve than (2.1). The vector $c_{1}$ can be obtained at the same time and at very little additional cost by solving the system $B c_{1}=e_{1}$. The computation of $\tau$ (n.b., $e_{1}{ }^{T} B^{-1} e_{1}$ is the first component of $c_{1}$ ) and $x$ entails a negligible amount of additional calculation. Note that once $c_{1}$ has been calculated, it can be saved and used to solve other systems of the form (2.1) with differing right-hand sides.

Concerning Gaussian elimination, suppose that at the $k$ th step an unacceptably small pivot element emerges. Then a solution of (2.1) may be obtained in the form $x^{T}=\left(x_{1}{ }^{(k) T}, x_{2}{ }^{(k) T}\right)$ as follows.

(1) Solve the system

$$
\left(\begin{array}{ll}
L_{11}^{(k)} & 0 \\
L_{21}^{(k)} & I
\end{array}\right)\left(\begin{array}{l}
b_{1}^{(k)} \\
b_{2}^{(k)}
\end{array}\right)=\left(\begin{array}{l}
b_{1} \\
b_{2}
\end{array}\right)
$$


(2) Set $B_{22}{ }^{(k)}=A_{22}{ }^{(k)}+\sigma_{k} e_{1} e_{1}{ }^{T}$, where $\sigma_{k}$ is chosen to make the pivot element acceptably large.

(3) Solve the systems

$$
B_{22}^{(k)} y_{2}^{(k)}=b_{2}^{(k)}, \quad B_{22}^{(k)} c_{1}^{(k)}=e_{1} .
$$

(4) Correct $y_{2}{ }^{(k)}$ to yield a solution of the system

$$
A_{22}^{(k)} x_{2}^{(k)}=b_{2}^{(k)} \text {. }
$$

(5) Solve the system

$$
U_{11}^{(k)} x_{1}^{(k)}=b_{1}^{(k)}-U_{12}^{(k)} x_{2}^{(k)} .
$$

This process can be repeated should an unacceptably small pivot be encountered in step 3 of the above algorithm; however, here the economics are not as favorable. The time considerations are roughly the same; each application of the technique requires the solution of an additional set of equations involving the matrix $B_{22}{ }^{(k)}$, a negligible increase over the Gaussian reduction of $B_{22}{ }^{(k)}$ itself. However, each $c_{1}{ }^{(k)}$ must be stored, and, since they are columns of inverse matrices, they need not be sparse, even when the original matrices are. Thus in applications involving large sparse matrices, the technique cannot be used too many times.

There remains the problem of choosing $\sigma_{k}$. It is clear that $\sigma_{k}$ must not be too large; for as $\sigma_{k}$ increases, $A_{22}{ }^{(k)}+\sigma_{k} e_{1} e_{1}{ }^{T}$ becomes a slight perturbation of the singular matrix $\sigma_{k} e_{1} e_{1}{ }^{T}$. A natural choice is to take $\sigma_{k}$ to be just large enough to dominate the elements in the first column of $A_{22}{ }^{(k)}$, which corresponds to partial pivoting in the elimination process. Since the value of the next pivot element can easily be computed, it may be desirable to alter $\sigma_{k}$ slightly, say multiply it by a factor of two, whenever cancellation would occur in the calculation of the next pivot element.

It is hardly necessary to add that our results hold also for the Crout and Doolittle variants of Gaussian elimination, for which the discussion above remains valid with some slight and obvious modifications.**

3. Error Analysis. The algorithm described in the last section must be implemented in finite precision arithmetic, and it is important to assess the effects of the resulting rounding errors on the solution. For simplicity, we shall first assume that a modification is made at the first stage of the elimination and drop the superscripts $(k)$. We shall determine conditions under which the computed solution $x$ has a residual $r=b-A x$ that is small. Note that, whatever the value of $r, x$ is the solution of the system

$$
(A+E) x=b,
$$

where $E=r x^{T} /\|x\|^{2}$ satisfies $\|E\| /\|A\|=\|r\| /\|x\|\|A\|$ in the Frobenius norm, defined by $\|A\|^{2}=\operatorname{trace} A^{T} A$. Thus a small residual implies that $x$, however inaccurate, is the solution of a slightly perturbed problem.

The following notation will be used in the error analysis. The symbol $\langle k\rangle$, called a relative counter, will stand generically for a quotient of the form

** This baroque variation on the famous theme of Laplace (il est aisé à voir) is due to Ostrowski [Arch. Rational Mech. Anal., v. 1, 1958, p. 241]. 


$$
\langle k\rangle=\left(1+\rho_{1}\right)\left(1+\rho_{2}\right) \cdots\left(1+\rho_{l}\right) /\left(1+\rho_{l+1}\right)\left(1+\rho_{l+2}\right) \cdots\left(1+\rho_{k}\right),
$$

where the numbers $\left|\rho_{i}\right|$ are uniformly bounded by some small quantity. We shall also use the notation \#k\# for the deviation of $\langle k\rangle$ from unity:

$$
\# k \#=1-\langle k\rangle \text {. }
$$

The symbol \#k\# will be called an absolute counter. We shall assume that the bounds on the $\rho_{i}$ in (3.1) and on the integer $k$ are so restricted that

$$
|\# k \#| \leqq k \cdot \epsilon \leqq .1
$$

for some number $\epsilon$ of approximately the same size as the bound on the $\left|\rho_{i}\right|$. If $x$ is a vector, then $x\langle k\rangle$ will denote the vector $\left(x_{1}\langle k\rangle, x_{2}\langle k\rangle, \cdots, x_{n}\langle k\rangle\right)^{T}$, where each appearance of the counter $k$ may stand for a different value.

The relative and absolute counters have the following easily verified properties:

$$
\langle k\rangle l\rangle=\langle k+l\rangle, \quad 1 /\langle k\rangle=\langle k\rangle
$$

and

$$
\langle k\rangle-\langle l\rangle=\# k+l \# .
$$

The usual backward error bounds for $t$-digit, base $\beta$, floating-point arithmetic (see, e.g., [3]) can be expressed in the form

$$
f l(a \circ b)=(a \circ b)\langle 1\rangle \quad(\circ=\times, \div)
$$

and

$$
f l(a \pm b)=a\langle 1\rangle \pm b\langle 1\rangle,
$$

where $\epsilon$ in (3.2) is of the order $\beta^{-t}$.

We turn now to the analysis of the effect of modifying the 1-1 element of $A$. All quantities will denote the computed values, with the exception of $B=A+\sigma e_{1} e_{1}{ }^{T}$. The first step is to solve the systems $B y=b$ and $B c_{1}=e_{1}$. We assume this is done stably so that the computed solutions satisfy

$$
B_{1} y \equiv\left(B+E_{1}\right) y=b \text { and } B_{2} c_{1} \equiv\left(B+E_{2}\right) c_{1}=e_{1},
$$

where $\left\|E_{i}\right\| \leqq \epsilon_{i}\|B\| \quad(i=1,2)$ for some small $\epsilon_{i}$. Note that the single rounding error made in forming $B$ from $A$ may be absorbed in the error matrices $E_{i}$.

The next step is to compute $\tau$. There is no rounding error in the computation of $c_{11}=e_{1}{ }^{T} B_{2}{ }^{-1} e_{1}$. Hence

$$
\tau=f l\left[\left(c_{11}-\sigma^{-1}\right)^{-1}\right]=\frac{\langle 1\rangle}{c_{11}\langle 1\rangle-\sigma^{-1}\langle 2\rangle}
$$

or

$$
\tau+\sigma\langle 3\rangle-\tau \sigma c_{11}\langle 3\rangle=0
$$

Finally one computes

$$
x=f l\left(y-\tau y_{1} c_{1}\right)=y\langle 1\rangle-\tau y_{1} c\langle 3\rangle .
$$

From this it follows that 


$$
\tau y_{1} c_{1}=y\langle 4\rangle-x\langle 3\rangle,
$$

whence from (3.2)

$$
|\tau|\left|y_{1}\right|\left|c_{1}\right| \mid \leqq 1.1(\|x\|+\|y\|)
$$

Now

$$
\begin{aligned}
r & =b-A x=b-\left(B-\sigma e_{1} e_{1}^{T}\right) x=b-\left(B_{2}-\sigma e_{1} e_{1}^{T}\right) x-E_{2} x \\
& =b-\left(B_{2}-\sigma e_{1} e_{1}^{T}\right)\left(y\langle 1\rangle-\tau y_{1} c_{1}\langle 3\rangle\right)-E_{2} x \\
& =b-B_{2} y\langle 1\rangle-E_{2} x+y_{1}\left(\tau+\sigma\langle 1\rangle-\tau \sigma c_{11}\langle 3\rangle\right) e_{1}+y_{1} \tau B_{2} c_{1} \# 3 \#
\end{aligned}
$$

the last equality following from the fact that

$$
B_{2} c_{1}\langle 3\rangle=B_{2} c_{1}+B_{2} c_{1} \# 3 \#=e_{1}+B_{2} c_{1} \# 3 \# \text {. }
$$

On subtracting zero in the guise of (3.4) from the term in parentheses in the last member of (3.6), we obtain from (3.3)

$$
r=b-B_{2} y\langle 1\rangle-E_{2} x+y_{1}\left(\sigma \# 4 \#-\tau \sigma c_{11} \# 6 \#\right) e_{1}+y_{1} \tau B_{2} c_{1} \# 3 \# .
$$

Hence

$$
\begin{aligned}
r= & b-B_{1} y+\left(E_{1}-E_{2}\right) y+B_{2} y \# 1 \#-E_{2} x \\
& +y_{1}\left(\sigma \# 4 \#-\tau \sigma c_{11} \# 6 \#\right) e_{1}+y_{1} \tau B_{2} c_{1} \# \# .
\end{aligned}
$$

Since $b-B_{1} y=0$, if we let

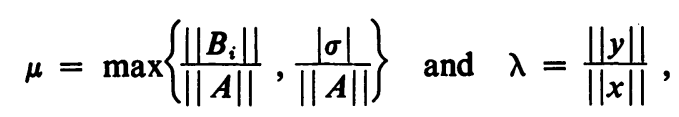

then from (3.2)

$$
\begin{aligned}
\frac{\|r\|}{\|A\|\|x\|} & \leqq\left(\epsilon_{1}+\epsilon_{2}\right) \lambda+\epsilon \mu \lambda+\epsilon_{2}+4 \epsilon \lambda \mu+6.6 \epsilon \mu(1+\lambda)+3.3 \epsilon \mu(1+\lambda) \\
& \leqq \epsilon_{2}+10 \epsilon \mu+\lambda\left(\epsilon_{1}+\epsilon_{2}+15 \epsilon \mu\right) .
\end{aligned}
$$

This result is quite satisfactory. For reasonable modifications of the pivot element, the number $\mu$ will be of order unity. Thus $\lambda$, the ratio of the sizes of the computed solutions of the equations $A x=b$ and $B y=b$, is the controlling factor. If $\lambda$ is large, that is if severe numerical cancellation occurs in the passage from $y$ to $x$, the result cannot be guaranteed to have a small residual. Note that this cannot happen if $B$ is well conditioned, whatever the condition of $A$. In any event, the condition is one that can be easily checked.

There remains one point to clear up. The modification step is only one part of the algorithm described in the last section, and we must show that this algorithm as a whole is stable. The usual rounding error analysis for triangular systems shows that the computed vectors $b_{1}{ }^{\left({ }^{k}\right)}$ and $b_{2}{ }^{(k)}$ satisfy

$$
\left(\begin{array}{ll}
L_{11}^{(k)}+F_{11} & 0 \\
L_{21}^{(k)}+F_{21} & I
\end{array}\right)\left(\begin{array}{l}
b_{1}^{(k)} \\
b_{2}^{(k)}
\end{array}\right)=\left(\begin{array}{l}
b_{1} \\
b_{2}
\end{array}\right),
$$

where $F_{11}$ and $F_{21}$ are small compared to $L_{11}{ }^{(k)}$ and $L_{21}{ }^{(k)}$. The results of this section 
imply that, if all has gone well, the computed vector $x_{2}{ }^{(k)}$ will satisfy

$$
\left(A_{22}^{(k)}+G_{22}\right) x_{2}^{(k)}=b_{2}^{(k)},
$$

where $G_{22}$ is small compared with $A_{22}{ }^{(k)}$. Since the solution for $x_{1}{ }^{(k)}$ amounts to no more than the completion of the solution of a triangular system, the computed vector $x$ satisfies

$$
\left(\begin{array}{cc}
U_{11}^{(k)}+G_{11} & U_{12}^{(k)}+G_{12} \\
0 & A_{22}^{(k)}+G_{22}
\end{array}\right)\left(\begin{array}{l}
x_{1}^{(k)} \\
x_{2}^{(k)}
\end{array}\right)=\left(\begin{array}{l}
b_{1}^{(k)} \\
b_{2}^{(k)}
\end{array}\right),
$$

where $G_{11}$ and $G_{22}$ are small compared with $U_{11}{ }^{(k)}$ and $U_{12}{ }^{(k)}$. Equations (3.7) and (3.8) can be combined in the usual way to show that the computed solution satisfies $(A+H) x=b$ where $H$ is small compared with $A$ (see for example [3, p. 108], in which the final bound must be supplemented by a factor of $\|L\|$ since no assumptions about pivoting strategy have been made).

Department of Computer Science

Carnegie-Mellon University

Pittsburgh, Pennsylvania 15213

1. Eli Hellerman \& Dennis C. Rarick, "The partitioned preassigned pivot procedure $\left(p^{\circ}\right), "$ in Sparse Matrices and their Applications, Donald J. Rose and Ralph A. Willoughby (Editors), Plenum Press, New York 1972, pp. 67-76.

2. A. S. HOUSEHOLDER, The Theory of Matrices in Numerical Analysis, Blaisdell, New York, 1964. MR 30 \#5475.

3. J. H. Wirknnson, Rounding Errors in Algebraic Processes, Prentice-Hall, Englewood Cliffs, N.J., 1963. MR 28 \#4661. 